Cahiers $d u$ MONDE RUSSE

\section{Cahiers du monde russe}

Russie - Empire russe - Union soviétique et États indépendants

$60 / 4 \mid 2019$

Varia

\title{
Kirill OSPOVAT, Terror and Pity. Aleksandr Sumarokov and the Theater of Power in Elizabethan Russia
}

\section{Rodolphe Baudin}

\section{(2) OpenEdition \\ 12 Journals}

Édition électronique

URL : https://journals.openedition.org/monderusse/11472

DOI : $10.4000 /$ monderusse. 11472

ISSN : $1777-5388$

Éditeur

Éditions de l'EHESS

\section{Édition imprimée}

Date de publication : 1 octobre 2019

Pagination : 819-822

ISBN : 978-2-7132-2797-4

ISSN : $1252-6576$

Référence électronique

Rodolphe Baudin, « Kirill OSPOVAT, Terror and Pity. Aleksandr Sumarokov and the Theater of Power in Elizabethan Russia », Cahiers du monde russe [En ligne], 60/4 | 2019, mis en ligne le 01 octobre 2019, consulté le 08 janvier 2023. URL : http://journals.openedition.org/monderusse/11472 ; DOI : https:// doi.org/10.4000/monderusse. 11472 


\section{Kirill OSPOVAT}

\section{Terror and Pity}

\section{Aleksandr Sumarokov and the Theater of Power in Elizabethan Russia}

Boston : Academic Studies Press, (« Imperial Encounters in Russian History »), 2016, $316 \mathrm{p}$.

Sortie en 2016, la monographie de Kirill Ospovat s'inscrit dans le renouveau des études consacrées au théâtre russe du XVIII ${ }^{\mathrm{e}}$ siècle. Visant à dépasser l'analyse de la simple dimension esthétique des textes, ce renouveau s'appuie sur les outils fournis par la sociologie historique des pratiques théâtrales et de leur réception, l'anthropologie politique et l'histoire des émotions. Consacré aux tragédies d'Aleksandr Sumarokov, Terror and Pity s'organise en trois parties. La première étudie l'histoire de la tragédie russe comme pratique de cour mettant en scène différents « scenarios de pouvoir » supposés édifier le public. Les deuxième et troisième sont respectivement consacrées à deux des tragédies principales de la première partie de la carrière de Sumarokov, Horev (1747) et Gamlet (1748). Cette structure tripartite, qui présente le contexte politique et intellectuel dans lequel Sumarokov rédigea ces deux pièces, avant de les analyser en détail, porte donc essentiellement sur le début du règne d'Elisabeth Petrovna (1741-1761). Elle est complétée par un épilogue consacré, au terme de ce règne de vingt ans, aux mécanismes culturels ayant présidé au " Miracle de la Maison de Brandebourg », c'est-à-dire la volte-face de Pierre III dans la guerre de Sept ans qui sauva Frédéric II du désastre militaire en 1762. Cet épisode de la politique étrangère russe est analysé ici comme l'application d'un scenario politique d'origine théâtrale, dont est également étudiée la réception à la cour de Russie.

Résumant en introduction l'apport des travaux des spécialistes du théâtre russe de l'époque d'Elisabeth Petrovna - de V. Vsevolodskij-Gerngross à G. GukovskijKirill Ospovat propose de les compléter grâce aux outils méthodologiques de $\mathrm{R}$. Wortman et $\mathrm{W}$. Benjamin, et présente le genre tragique comme un langage fait d'allégories et d'allusions politiques exprimant les principes idéologiques de la société de cour et les tensions émotionnelles organisant son existence sociale. Grâce à cette approche, l'auteur de Terror and Pity vise à révéler l'affinité fondamentale reliant la poétique de la tragédie classique aux mécanismes discursifs du pouvoir dans la Russie autocratique. Suscitant tour à tour la terreur et la pitié, les tragédies de Sumarokov présentent des scénarios reflétant l'exercice du pouvoir absolutiste russe, en même temps qu'elles lui fournissent des modèles littéraires amenés à en légitimer les pratiques et à en modeler une réception émotionnelle adéquate par le public de la cour, considéré comme une représentation symbolique du corps politique. Ces scénarios avaient pour but de renforcer la souveraineté des autocrates russes sur leurs sujets, notamment lorsque celle-ci avait été établie dans les circonstances contestables des « révolutions de palais ». Leur étude montre comment la souveraineté tirait sa légitimité d'actes de violence extraordinaires, qui nourrissaient plutôt qu' ils n'affaiblissaient le charisme du monarque, ainsi que 
le constate Kirill Ospovat en s'appuyant sur les travaux de M. Weber, C. Schmitt et M. Foucault.

La lecture de Horev offerte par le chapitre premier commence par un rappel de l'importance de l'amour pour Sumarokov, poète " tendre » affublé du surnom de " Racine russe ». Appliquée à la tragédie, la thématique amoureuse devient allégorie du pouvoir et de la souveraineté de celui qui suscite l'amour sur celui qui tombe amoureux. Complément à l'amour, le mariage, souvent utilisé comme métaphore dans les relations internationales, notamment entre la Russie et la Suède en 1743, devient une allégorie de la réconciliation entre liberté (du choix) et captivité (des sujets) et est appelé à contenir la violence de l'exercice de la souveraineté en lui substituant la promesse de la paix civile. Central dans la tragédie pastorale diffusée en Russie par le succès du Pastor fido de Guarini, l'amour suppose la séduction, dont les contemporains notent l'utilisation par Elisabeth Petrovna pour assurer le succès de son coup d'État. D'abord secret et illégal, comme tout projet de révolution de palais, l'amour est ensuite sanctionné divinement dans la tragédie pastorale et reçoit habituellement à cet égard la bénédiction des parents des jeunes amants. La violation de cette convention de l'assentiment final par Sumarokov dans Horev permet cependant au dramaturge d'aborder un autre sujet, en écho aux préoccupations politiques de la cour russe : la peur du complot. Prévenu par le boyard Stalverh d'une supposée conspiration tramée par Horev, Kij, souverain de Kiev, reflète la position d'Elisabeth Petrovna, menacée par un possible coup d'État contraire, visant au rétablissement d'Anna Leopoldovna. En cela, la tragédie reflète l'inquiétude qui agitait, au début des années 1740, la cour russe, sous l'influence de la lecture des réflexions sur les coups d'État de Machiavel et de Saovedra Fajardo. Comme l'explique Kirill Ospovat, la mise en garde de Stalverh rappelait également celle d'Artemij Volynskij à Anna Leopoldovna et ses accusations, les soupçons ultérieurs portés contre Münnich et Natal'ja Lopuhina lors des procès politiques de 1742-1743. Se comportant comme Elisabeth Petrovna, Kij doute, mais punit malgré tout, dans un geste appelé à incarner sa souveraineté, qui illustre la théâtralité de la répression dans l'esthétique de l'absolutisme. Double de Lopuhina, l'amante de Horev Osnel' da est innocente, mais punie, suscitant la compassion de son juge Kij, dans un scénario qui le présente, comme Elisabeth Petrovna, tout à la fois comme tyran meurtrier et monarque bienveillant. Ce double statut, tout en rappelant sa puissance, consolide le lien émotionnel du souverain avec le public, via le partage d'une expérience commune de la pitié.

Objet du deuxième chapitre, Gamlet fut, comme son titre l'indique, librement adapté de Shakespeare, que Sumarokov découvrit dans sa traduction française. Prolongeant les analyses de M. Levitt sur la pièce, Kirill Ospovat étudie la tragédie comme le récit d'un rétablissement de la souveraineté véritable grâce à une intervention divine, dans le but d'éclairer de manière correcte pour la cour la signification du coup d'État de novembre 1741. Dans cette perspective, l'usurpation de Polonius/Polonij est présentée comme une métaphore de celle des Braunschweig et le fantôme du père d'Hamlet, dont Sumarokov remplace l'apparition par une description de rêve, comme l'image de Pierre I $^{\text {er }}$ exigeant le rétablissement de 
Gamlet/Elisabeth Petrovna sur le trône. Contrairement à son prototype britannique, Gamlet reprend le pouvoir usurpé grâce à sa capacité à susciter l'émerveillement et l'admiration du public, que Kirill Ospovat, en s'appuyant sur Weber, interprète en termes de charisme. Présentée comme une rébellion, la récupération du pouvoir par Gamlet apparaît néanmoins comme légitime grâce à la sanction divine apportée par l'image du fantôme. Le succès ne vient toutefois couronner l'entreprise qu'après que Gamlet a surmonté l'indécision de son modèle shakespearien, nourri par une mélancolie que Kirill Ospovat analyse comme le régime émotionnel caractéristique du souverain dans l'Angleterre du XVII ${ }^{\mathrm{e}}$ siècle et la Russie du XVIII ${ }^{\mathrm{e}}$ siècle. Incapacitante chez Hamlet, la mélancolie se révèle masque chez Gamlet, et capacité machiavélienne à attendre le moment politiquement opportun, dans une stratégie de conquête du pouvoir attribuée postérieurement par les contemporains à Elisabeth Petrovna et rappelant la construction en cinq actes de la tragédie classique. Parvenu au pouvoir, Gamlet punit Klavdij et Polonij comme Elisabeth Petrovna punit Ostermann et Münnich, représentés comme l'incarnation de la malice et de l'intrigue, comme l'était le Polifonte du Mérope de Voltaire, hypotexte complémentaire utilisé par Sumarokov en plus de Hamlet. Comme l'explique Kirill Ospovat, les deux coupables de confession protestante sont également présentés comme des instruments du démon, rappelant en cela les accusations portées contre une autre incarnation de l'homme de cour comploteur, Dmitrij Golicyn, jugé et condamné en 1737. Le dénouement de la tragédie met en scène le pardon de Polonij, comme Elisabeth Petrovna pardonna en 1742 à Ostermann et Münnich, dont l'exécution fut commuée en peine d'exil. Dans les deux cas, l'exercice de la clémence mis en scène faisait partie d'un scénario de pouvoir inspiré par la Clémence de Titus (1734) de Métastase et nourri de l'idée que le pardon n'est pas un aveu de faiblesse, mais bien au contraire un exercice de domination absolue. Tout comme le supplice public d'Ostermann et Münnich et son heureux dénouement, la pièce de Sumarokov était supposée susciter chez le public la terreur et la pitié, produisant la catharsis aristotélicienne permettant la purge des passions et la renégociation d'un accord de gouvernement entre sujets fidèles et monarque triomphant. Cette catharsis était en outre inscrite métatextuellement dans la pièce, à travers la mise en scène par la tragédie du repentir de Gertruda, modèle de réception émotionnelle du texte offert au public russe contemporain.

Ce scénario de clémence comme démonstration de la puissance politique est au centre de l'épilogue de Terror and Pity, où il sert à l'auteur à présenter sous un éclairage nouveau la volte-face russe vis-à-vis de Frédéric II lors de l'accession de Pierre III au trône en 1762. Bien loin d'être le caprice d'un prussomane, ce revirement est analysé ici comme l'adhésion à un scénario de pouvoir proposé à la cour russe par la lecture de l'Epître à $M$. de Voltaire (1752) de Frédéric II. Vivant son rôle de souverain comme un rôle de théâtre et voyant Marie-Thérèse d'Autriche en Athalie, Frédéric se compare à Porus, roi indien défait par Alexandre dans Alexandre le Grand (1665) de Racine et Alessandro nell'Indie (1729) de Métastase. Si le roi de Prusse utilise le rôle de Porus pour susciter le zèle patriotique de ses sujets, Pierre III, qui connaissait tant l'œuvre de Frédéric que celle de Métastase, 
endosse quant à lui celui d'Alexandre, qui le mène à faire preuve de clémence. C'est ainsi non par faiblesse, mais pour performer un geste affirmant sa souveraineté que Pierre sauve Frédéric, dans une stratégie censée augmenter son charisme et subjuguer son ennemi vaincu autant que ses propres sujets. En ce sens, le revirement de Pierre III s'inscrit dans la lignée des autres gestes de clémence que sont le Manifeste sur les libertés de la noblesse et l'abolition de la Chancellerie secrète. Si les contemporains furent bel et bien émerveillés par la clémence de Pierre III vis-à-vis de Frédéric et de la noblesse russe, l'empereur se montra finalement incapable de maintenir les règles de son engagement performatif avec le public et son geste fut perçu comme une violation du corps politique.

De fait, explique Kirill Ospovat en conclusion, Pierre III n'appartenait visiblement pas au nombre des souverains acteurs sachant se comporter conformément aux matrices émotionnelles et politiques fournies par la tragédie et alterner gestes de terreur et de pitié pour augmenter leur charisme et renforcer à leur profit l'adhésion du public, au double sens de public théâtral et de nation.

Comme le suggère ce long résumé de l'ouvrage de Kirill Ospovat, Terror and Pity est une étude profondément novatrice et d'une exceptionnelle richesse. Dans un va-et-vient d'une étourdissante habileté entre théorie contemporaine du politique et sources historiques variées - des traités politiques occidentaux de la première Modernité aux témoignages publics ou privés sur la vie de cour dans la Russie des années 1730-1740, en passant par le répertoire théâtral et opératique européen - l'auteur propose une approche foncièrement renouvelée de l'œuvre de Sumarokov, que l'historiographie cantonnait jusque-là trop souvent à un rôle d'agent de l'importation du classicisme ou de certains aspects du discours des Lumières en Russie. En révélant la capacité des textes tragiques à exprimer la violence cachée des rapports de pouvoir entre souverains et sujets, Kirill Ospovat explique non seulement bien des aspects, jugés jusqu'ici comme autant de maladresses, de l'intrigue de ses pièces, mais il révèle également un degré insoupçonné d'engagement émotionnel du public russe avec la littérature du classicisme. Supposée tout à la fois refléter, entretenir et expliquer aux courtisans de Saint-Pétersbourg les émotions qu'ils vivaient, et au premier rang desquelles se trouvait la terreur, cette littérature était l'instrument indispensable de la mise en scène de la grandeur politique, dont ils étaient invités à être les spectateurs disciplinés.

Rodolphe Baudin

Sorbonne Université 